\title{
Factors associated with prolonged length of stay for elective hepatobiliary and neurosurgery patients: a retrospective medical record review
}

Siu Yin Lee', Soo-Hoon Lee ${ }^{2}$, Jenny H. H. Tan ${ }^{3}$, Howard S. L. Foo ${ }^{4}$, Phillip H. Phan ${ }^{5^{*}}$ (D, Alfred W. C. Kow ${ }^{1}$, Sein Lwin ${ }^{1}$, Penelope M. Y. Seah ${ }^{1}$ and Siti Zubaidah Mordiffi ${ }^{1}$

\begin{abstract}
Background: Patients with prolonged length of hospital stay (LOS) not only increase their risks of nosocomial infections but also deny other patients access to inpatient care. Hepatobiliary (HPB) malignancies have some of highest incidences in East and Southeast Asia and the management of patients undergoing HPB surgeries have yet to be standardized. With improved neurosurgery techniques for intracranial aneurysms and tumors, neurosurgeries (NS) can be expected to increase. Elective surgeries account for far more operations than emergencies surgeries. Thus, with potentially increased numbers of elective HPB and NS, this study seeks to explore perioperative factors associated with prolonged LOS for these patients to improve safety and quality of practice.
\end{abstract}

Methods: A retrospective cross-sectional medical record review study from January 2014 to January 2015 was conducted at a 1250-bed tertiary academic hospital in Singapore. All elective HPB and NS patients over 18 years old were included in the study except day and emergency surgeries, resulting in 150 and 166 patients respectively. Prolonged LOS was defined as above median LOS based on the complexity of the surgical procedure. The predictor variables were preoperative, intraoperative, and postoperative factors. Student's t-test and stepwise logistic regression analyses were conducted to determine which factors were associated with prolonged LOS.

Results: Factors associated with prolonged LOS for the HPB sample were age and admission after 5 pm but for the NS sample, they were functional status, referral to occupational therapy, and the number of hospital-acquired infections.

Conclusion: Our findings indicate that preoperative factors had the greatest association with prolonged LOS for HPB and NS elective surgeries even after adjusting for surgical complexity, suggesting that patient safety and quality of care may be improved with better pre-surgery patient preparation and admission practices.

Keywords: Prolonged length of stay, Elective surgery, Preoperative, Intraoperative, Postoperative care, Ambulatory services

\footnotetext{
* Correspondence: pphan@jhu.edu

${ }^{5}$ Johns Hopkins University, 100 International Drive, Baltimore, MD 21202, USA

Full list of author information is available at the end of the article
} 


\section{Background}

Length of stay (LOS) is an important measure of resource utilization as patients with prolonged LOS disproportionately account for the consumption of more hospital resources [1]. Prolonged LOS, which is defined as inpatient stay that exceeds the expected LOS for a certain procedure [2], unnecessarily utilizes hospital beds, contributing to capacity shortage. Inpatient bed shortage delays elective admissions and increases emergency department boarding, which denies critically ill patients timely access to treatment, as inpatient care conventionally begins only after the patient arrives at the assigned inpatient ward [3, 4]. Patients with prolonged LOS are also at higher risks of nosocomial infections and unplanned readmissions $[5,6]$. In short, prolonged LOS disadvantages both hospitals and patients [7].

Surgical services represent approximately $30 \%$ of all hospital expenses [8] and elective surgeries are far more common than emergency operations [9]. Unlike emergency admissions where it is difficult for hospital administrators to influence pre-admission factors, knowing the factors that increase LOS in elective surgeries can allow hospital staff to proactively manage the delivery of care and demand for beds [10]. While past studies have explored the factors associated with prolonged LOS after cardiac [11], laparoscopic cholecystectomy [12-14], and spine $[15,16]$ surgeries, no studies have explored the factors that lead to prolonged LOS in hepatobiliary (HPB) and neurosurgeries (NS). HPB malignancies, liver tumors, and other liver diseases have the highest incidences in Asia and Southeast Asia compared to the rest of the world due to widespread hepatitis B virus infections [17]. Surgery remains the only option for treatment. However, HPB procedures are variable and their care management practices are not standardized [18]. Consequently, the LOS for HPB surgical patients varies widely. For NS, the demand for such surgeries is expected to increase because of improved surgical procedures in craniotomy and cranioplasty for intracranial aneurysms, brain tumors, and other cerebrovascular diseases as well as aging populations and changes in lifestyle in developed countries, including Asia [19]. By explicating factors associated with prolonged LOS for HPB and NS elective surgeries, hospitals can design better patient management services [20]. In sum, studying these two types of high demand elective surgical procedures is more likely to lead to meaningful reductions in the overuse of bed capacity.

Multiple factors at the patient and systems level have been associated with LOS in a variety of disease settings. For example, common preoperative patient factors associated with prolonged LOS are the patient's demographics and presenting conditions at the time of admission [21]. Studies have shown that age, gender, functional status, diagnostic or illness factors, and the major procedure group for surgical patients influenced LOS [2, 3, 12-14, 22]. Specifically, age was related to prolonged LOS for patients undergoing aortic surgery [23]. In general, elderly patients undergoing a major surgery face higher risks of bleeding and other physiological dysfunctions that may slow recovery and contribute to prolonged LOS [24]. Seicean et al. [15] found that elective spine surgery patients with higher bodymass index (BMI) reported poorer outcomes. The Braden score on admission, which identifies patients at higher risk for pressure ulcers, was found to predict prolonged LOS for patients undergoing liver transplantation [25], while malnutrition on admission slows recovery [26]. Patients who are frail and cannot ambulate independently have a low functional status score and were reported to have prolonged LOS following elective lumbar spine and arterial vascular surgeries [27, 28]. Comorbidity also predicted prolonged LOS [29]. Gruskay et al. [16] found that the total number of comorbidities predicted prolonged LOS after elective lumbar surgery. Likewise, the number of abnormal laboratory results (labs) indicate the severity of patients' health status, which influences how fast they will recover after surgery. Fall risk is measured by assessing the patient's level of confusion, dizziness, altered elimination, and difficulty with mobility at admission [30, 31]. Preoperative fall risks have been found to predict postoperative falls, functional decline, and surgical complications, all of which prolonged LOS [32]. Occupational therapy (OT) improves walking capacity, muscle strength, flexibility, balance, and endurance so patients can quickly return to active lifestyles $[33,34]$. However, patients need a minimum capacity to do OT, and preoperative physiotherapy (PT) has been shown to improve exercise tolerance for patients undergoing lung transplantation [35].

Preoperative hospital administrative system factors that contribute to LOS include delays in investigation and procedures [36] as well as the day and time of admission [37]. For example, patients admitted during weekends, on the eve of public holidays, or after $5 \mathrm{pm}$ often face delays in the initial physical examination and diagnostic tests due to diminished availability of staff and expertise. In general, perioperative patient care after office hours is limited by a reduced clinical crew. Consequently, the day and time of admission have been associated with increased LOS, preventable adverse events, and mortality of patients with pulmonary embolism and myocardial infarction $[37,38]$.

Intraoperative variables that affect LOS include the duration of preoperative preparation [39] and operation time [2]. For example, for patients undergoing surgery for endocrine tumor, the duration of preoperative preparation for anesthesiologic management, fluid management, 
medications involving anti-hypertensive medications, alpha, beta, or calcium channel blockers, and antibiotic prophylaxes, were associated with prolonged LOS [39]. Delays or conflicts in preoperative preparation may lead to a cancellation of the surgery, which further increases LOS. Chang et al. [23] found that operations lasting more than $5 \mathrm{~h}$, which may be related to intraoperative blood loss, were associated with prolonged LOS for patients undergoing aortic surgery.

Postoperative variables that affected LOS include the number of complications after surgery and hospitalacquired infections (HAI) [2, 23, 40, 41]. For example, complications such as urinary tract infection and pneumonia led to prolonged LOS following cervical discectomy and fusion [41]. Postoperative treatment for wound or surgical site infections [23], polypharmacy [40, 42] where multiple drug consumption increased the risk of drug interactions and adverse reactions, as well as use of dialysis, ventilator support, and catheter insertions [3] also contributed to LOS. Other hospital acquired nosocomial infections include infections from clostridium difficile colitis [43] or methicillin-resistant Staphylococcus aureus [6]. Research has also shown that discharge planning affects LOS, where discharge to a nursing home was associated with prolonged LOS by $89 \%$ for surgical patients compared to being discharged to home $[3,36]$.

In sum, there is broad literature to suggest that multifactor perioperative pathways, excluding the operation procedure, have significant impact on prolonged LOS. However, current studies exploring such factors have only focused on partial perioperative pathways. For example, Freitas et al. [7], Reponen et al. [44], and Lukasiewicz et al. [21] focused on the patients' preoperative clinical conditions, Morimoto et al. [13] investigated preoperative and intraoperative clinical factors, while Collins et al. [2] considered intraoperative and postoperative factors. These studies could not rule out confounding factors from the partial pathway that could otherwise be non-significantly related to prolonged LOS if the entire perioperative pathway was considered. In this study, we examined perioperative factors in the entire surgical pathway. By assessing the simultaneous impact of these factors on prolonged LOS, hospitals can set reasonable preoperative expectations for patients and the healthcare team, establish efficient operative protocols, or plan postoperative treatment care and improve discharge planning for patients. Our approach is more likely to yield a comprehensive surgical services protocol for the entire perioperative care continuum for elective HPB and NS patients.

The objective of this study is to assess the association between preoperative, intraoperative, and postoperative factors on prolonged LOS after adjusting for surgical complexity for a sample of elective HPB and NS patients. Since surgeries are more susceptible to the risk of adverse events than non-surgical procedures [45, 46] and elective surgeries outnumber emergency procedures, we contribute to the literature on surgical safety and quality of care with greater impact for potential policy interventions that influence LOS. In the next section, we describe the setting and design of the study, followed by a description of the patient population, the data collection process, the variables, and the statistical analyses. We then report and discuss the results. We conclude with a discussion on the applications of the findings.

\section{Methods \\ Design and setting}

We conducted a retrospective cross-sectional medical record review of elective HPB and NS patients admitted between January 2014 and January 2015 to a 1250-bed tertiary academic center in Singapore, which was the first to receive the Joint Commission International accreditation in the country (http://www.nuhs.edu.sg/about-us/corporate-profile.html). Our study site mirrors the challenge of bed shortages in public hospitals in urban environments as well as incidences of HPB malignancies and demand for NS due to an aging population [47].

\section{Participants}

All patients over 18 years old who were admitted between January 2014 and January 2015 for elective HPB and NS procedures via the hospital's specialist outpatient clinics were included in the study. Patients who were admitted for emergency surgeries and day surgeries, such as repairs of inguinal hernia, anal fissure, appendectomy, and cholecystectomy, were excluded from the study. The samples for this study were $150 \mathrm{HPB}$ and 166 NS patients.

\section{Data collection}

One nurse administrator and one operations administrator, who were not involved in surgeries, extracted preoperative, intraoperative, and postoperative information from the medical records with the assistance of five registered nurses. This study was IRB approved by the National Healthcare Group Domain-Specific Review Board (DSRB 2015/00229).

\section{Measures}

The outcome variable in this study was prolonged LOS. The Singapore Ministry of Health provides a Table of Surgical Procedures (TSP), equivalent to the American Society of Anesthesiologists (ASA) class codes, which is an exhaustive list of surgical procedures ranked by complexity in ascending order from $1 \mathrm{~A}$ to $7 \mathrm{C}$ for insurance claims [48]. We defined prolonged LOS as the days of hospital stay above the median LOS of the TSP, as defined by Collins et al. [2] and Morimoto et al. [13], 
as shown in Additional file 1. The complexity of surgery influences prolonged LOS and so shortened LOS is defined as LOS below the median LOS of the TSP.

Coding of the predictor variables is provided in Additional file 2. Since prolonged LOS for HPB and NS have not been previously studied, we looked to the literature on other types of surgeries to determine the appropriate predictor variables on prolonged LOS. Preoperative factors found in other types of surgeries, as discussed earlier, included: (1) patients' demographics (age, gender, and race), (2) patients' presenting conditions at admission (BMI, Braden Scale [49], Nutrition Score [50], functional status measured by the Katz Index [51], smoking history, number of comorbidities, number of abnormal labs, and number of fall risks), and (3) hospital administrative system factors (weekend admission, admission after 5 pm, referrals to PT and OT, and number of delays in diagnostic tests and appointments).

Intraoperative factors found in other types surgeries that influenced LOS, as discussed earlier, included surgical factors related to (1) preparation duration, which is the time interval between the patient's entry into the operating theatre and incision based on the TSP, (2) operation duration, which is the time interval between incision to closure based on the TSP, and (3) operation ending after $5 \mathrm{pm}$.

Finally, postoperative factors found in other types surgeries that influenced LOS, as discussed earlier, included: (1) post-surgery inpatient factors (number of insertions, number of HAI, and number of medications) and (2) discharge planning (discharged after $5 \mathrm{pm}$ ).

\section{Statistical analysis}

As our study included many predictor variables with small sample sizes, we analyzed the data in two steps. First, we conducted Student's t- and $\chi^{2}$-tests on the continuous and dichotomous variables respectively, to identify factors that statistically differentiated between prolonged and shortened LOS. Next, we used these factors in a stepwise logistic regression analysis to derive the predictive model of prolonged LOS. We conducted a backward logistic regression analysis to verify the results. The statistical analyses were performed using SPSS version 23 [52] and statistical significance was assumed at $p<0.05$ throughout this study.

\section{Results}

The data reports that the median LOS for both the HPB and NS samples was 6 days. The interquartile range (IQR), which indicates the 25 th and 75 th percentile values, for the HPB sample was 2-8 days while the IQR for the NS sample was 4-9 days. The mean ( \pm standard deviation) LOS for the HPB and NS samples were 7.18 $( \pm 8.01)$ and $10.99( \pm 21.02)$ days respectively.
Table 1 reports the factors that significantly differentiated between prolonged and shortened LOS for the HPB sample. Among the preoperative factors, they were age and admission after $5 \mathrm{pm}$; among the intraoperative factors, they were preparation duration, operation duration, and operations ending after $5 \mathrm{pm}$; and among the postoperative factors, they were the number of insertions and the number of HAI.

Table 2 reports the factors that significantly differentiated between prolonged and shortened LOS for the NS sample. Among the preoperative factors, they were age, gender, functional status, the number of abnormal labs, the number of fall risks, admission after $5 \mathrm{pm}$, and referrals to PT and OT. Among the intraoperative factors, it was operation duration; and among the postoperative factors, they were the number of HAI, the number of medications, and discharged after $5 \mathrm{pm}$.

In the logistic regression models, Table 3 reports that only preoperative factors (age and admission after $5 \mathrm{pm}$ ) predicted prolonged LOS in the HPB sample after adjusting for surgical complexity. Table 4 reports that preoperative factors (functional status and referrals to OT) predicted prolonged LOS in the NS sample after adjusting for surgical complexity. The only postoperative factor to predict prolonged LOS in the NS sample was the number of HAI.

\section{Discussion}

Among the preoperative, intraoperative, and postoperative factors that statistically differentiated between prolonged and shortened LOS in our samples, the logistic regression model reports that preoperative factors in the surgical pathway related to patient factors were the most predictive of prolonged LOS. Specifically, age was associated with prolonged LOS for the HPB sample, while functional status and referrals to OT were associated with prolonged LOS for the NS sample. In line with Chang's [23] study on aortic surgery, age was related to prolonged LOS. Elderly patients face physiological dysfunctions from major surgeries that may slow recovery and delay hospital discharge [24]. Age is also a multifactorial demographic characteristic that is related to functional status [27], comorbidities [24, 42], polypharmacy [42], and postoperative complications [23].

For the NS sample, patient factors predictive of prolonged LOS from the logistic regression were functional status and referrals to OT. Similar to Partridge's [28] study on arterial vascular surgery, our data showed that low functional status and frail patients who needed OT had prolonged LOS. Our results may suggest that physiologically vulnerable patients may need additional care plans to improve their walking capacity, muscle strength, flexibility, balance, or endurance, thus prolonging LOS. 
Table 1 Student's t- or $x^{2}$-tests of differences between below and above median LOS for predictors in the HPB Sample

\begin{tabular}{|c|c|c|c|}
\hline Means or Percentages & At Below LOS Median of the TSP & At Above LOS Median of the TSP & $p$-value \\
\hline \multicolumn{4}{|l|}{ Preoperative Factors: } \\
\hline Age \pm s.d. & $55.54 \pm 14.29$ & $64.62 \pm 10.97$ & $<0.001$ \\
\hline Gender: Male (n) & $53.00 \%(35)$ & $65.20 \%(43)$ & 0.11 \\
\hline Race: Chinese (n) & $60.60 \%(40)$ & $57.60 \%(38)$ & 0.43 \\
\hline Unhealthy BMI (n) & $67.40 \%(29)$ & $65.20 \%(15)$ & 0.53 \\
\hline Braden Scale \pm s.d. & $22.73 \pm 0.93$ & $21.96 \pm 2.12$ & 0.06 \\
\hline Nutrition Score \pm s.d. & $0.36 \pm 0.91$ & $0.73 \pm 1.40$ & 0.18 \\
\hline Functional Status \pm s.d. & $0.26 \pm 0.91$ & $0.88 \pm 1.73$ & 0.06 \\
\hline Smoking History (n) & $15.60 \%(7)$ & $17.90 \%(5)$ & 0.52 \\
\hline Number of Comorbidities \pm s.d. & $1.39 \pm 1.10$ & $1.75 \pm 1.21$ & 0.18 \\
\hline Number of Abnormal Labs \pm s.d. & $2.49 \pm 1.68$ & $3.23 \pm 1.58$ & 0.08 \\
\hline Number of Fall Risks \pm s.d. & $0.05 \pm 0.21$ & $0.15 \pm 0.36$ & 0.13 \\
\hline Weekend Admissions (n) & $9.10 \%(6)$ & $9.10 \%(6)$ & 0.99 \\
\hline Admissions After 5 pm (n) & $18.20 \%(12)$ & $50.00 \%(33)$ & $<0.001$ \\
\hline Referral to OT (n) & $9.10 \%(4)$ & $14.80 \%(4)$ & 0.36 \\
\hline Referral to PT (n) & $18.20 \%(8)$ & $29.60 \%(8)$ & 0.20 \\
\hline Number of Delays \pm s.d. & $1.11 \pm 1.29$ & $2.19 \pm 3.76$ & 0.66 \\
\hline \multicolumn{4}{|l|}{ Intraoperative Factors: } \\
\hline Preparation Duration \pm s.d. & $1: 02: 51 \pm 0: 26: 16$ & $1: 14: 35 \pm 0: 29: 44$ & 0.02 \\
\hline Operation Duration \pm s.d. & $2: 53: 09 \pm 2: 09: 03$ & $3: 48: 42 \pm 2: 36: 39$ & 0.03 \\
\hline Operation Ends After 5 pm (n) & $19.70 \%(13)$ & $40.90 \%(27)$ & 0.01 \\
\hline \multicolumn{4}{|l|}{ Postoperative Factors: } \\
\hline Number of Insertions \pm s.d. & $1.89 \pm 2.16$ & $1.18 \pm 1.77$ & 0.02 \\
\hline Number of $\mathrm{HAl} \pm$ s.d. & $0.20 \pm 1.21$ & $0.48 \pm 0.85$ & 0.01 \\
\hline Number of Medications \pm s.d. & $0.18 \pm 0.45$ & $0.44 \pm 0.85$ & 0.46 \\
\hline Weekend Discharge (n) & $37.90 \%(25)$ & $28.80 \%(19)$ & 0.18 \\
\hline Discharged After 5 pm (n) & $12.10 \%(8)$ & $19.70 \%(13)$ & 0.17 \\
\hline
\end{tabular}

LOS length of stay

$H P B$ hepatobiliary

TSP table of surgical procedure

s.d standard deviation

$B M I$ body-mass index

Unhealthy BMI BMI outside the 18.5-23 range

OT occupational therapy

PT physiotherapy

$H A l$ hospital-acquired infections

The implications of knowing that age, functional status, and frailty needing OT may be associated with prolonged LOS for HPB and NS patients are that hospitals could implement evidence-based policies to prepare such patients for surgery to increase patient safety and reduce LOS. For example, hospitals could consider adopting preoperative protocols such as home visits and enhanced recovery after surgery (ERAS) protocols [53] to prepare elderly or frail patients for upcoming elective surgeries [54]. By making patients more aware of their recovery pathways, such protocols empower patients and their families to take responsibility (e.g., for early and frequent ambulation) for their own well-being.
The logistic regression model for the HPB sample indicates that among systems factors, admission after $5 \mathrm{pm}$ was associated with prolonged LOS, in line with Earnest's [37] study. Admission to the hospital for surgery after $5 \mathrm{pm}$ is an indicator of an operational inefficiency since no elective surgeries occurred at night at this academic center. Admission after $5 \mathrm{pm}$ not only added another day of hospital stay but patients also face delays in diagnostic tests as well as limited inpatient care due to the diminished availability of staff and expertise at night. This finding may suggest that hospitals could consider establishing a policy of not admitting patients for elective surgeries after $5 \mathrm{pm}$ to avoid an additional 
Table 2 Student's t- or $X^{2}$-tests of differences between below and above median LOS for predictors in the NS sample

\begin{tabular}{|c|c|c|c|}
\hline Means or Percentages & At Below LOS Median of the TSP & At Above LOS Median of the TSP & p-value \\
\hline \multicolumn{4}{|l|}{ Preoperative Factors: } \\
\hline Age \pm s.d. & $46.56 \pm 17.38$ & $52.87 \pm 15.91$ & 0.03 \\
\hline Gender: Male (n) & $31.80 \%(21)$ & $47.40 \%(37)$ & 0.04 \\
\hline Race: Chinese (n) & $62.10 \%(41)$ & $67.90 \%(53)$ & 0.29 \\
\hline Unhealthy BMI (n) & $78.80 \%(26)$ & $64.50 \%(20)$ & 0.16 \\
\hline Braden Scale \pm s.d. & $22.42 \pm 1.23$ & $21.39 \pm 2.79$ & 0.06 \\
\hline Nutrition Score \pm s.d. & $0.16 \pm 0.50$ & $0.38 \pm 0.92$ & 0.42 \\
\hline Functional Status \pm s.d. & $0.14 \pm 0.68$ & $1.27 \pm 1.91$ & $<0.001$ \\
\hline Smoking History (n) & $10.50 \%(4)$ & $12.50 \%(5)$ & 0.53 \\
\hline Number of Comorbidities \pm s.d. & $0.95 \pm 0.93$ & $1.24 \pm 1.02$ & 0.19 \\
\hline Number of Abnormal Labs \pm s.d. & $1.65 \pm 1.10$ & $2.75 \pm 1.35$ & $<0.001$ \\
\hline Number of Fall Risks \pm s.d. & $0.32 \pm 0.85$ & $0.57 \pm 0.70$ & 0.02 \\
\hline Weekend Admissions (n) & $34.80 \%(23)$ & $33.30 \%(26)$ & 0.49 \\
\hline Admissions After 5 pm (n) & $33.30 \%(22)$ & $54.70 \%(41)$ & 0.01 \\
\hline Referral to OT (n) & $11.10 \%(4)$ & $32.40 \%(11)$ & 0.03 \\
\hline Referral to PT (n) & $13.90 \%(5)$ & $35.30 \%(12)$ & 0.04 \\
\hline Number of Delays \pm s.d. & $1.40 \pm 1.73$ & $1.39 \pm 1.69$ & 0.60 \\
\hline \multicolumn{4}{|l|}{ Intraoperative Factors: } \\
\hline Preparation Duration \pm s.d. & $1: 07: 43 \pm 0: 24: 03$ & $1: 15: 08 \pm 0: 26: 48$ & 0.09 \\
\hline Operation Duration \pm s.d. & $1: 40: 52 \pm 0: 58: 15$ & $2: 27: 16 \pm 1: 55: 03$ & $<0.001$ \\
\hline Operation End After 5 pm (n) & $15.20 \%(10)$ & $17.90 \%(14)$ & 0.41 \\
\hline \multicolumn{4}{|l|}{ Postoperative Factors: } \\
\hline Number of Insertions \pm s.d. & $0.85 \pm 1.04$ & $1.18 \pm 1.35$ & 0.24 \\
\hline Number of $\mathrm{HAl} \pm$ s.d. & $0.05 \pm 0.23$ & $0.55 \pm 0.83$ & $<0.001$ \\
\hline Number of Medications \pm s.d. & $0.08 \pm 0.36$ & $0.56 \pm 0.82$ & $<0.001$ \\
\hline Weekend Discharge (n) & $37.90 \%(25)$ & $24.40 \%(19)$ & 0.06 \\
\hline Discharged After 5 pm (n) & $18.20 \%(12)$ & $36.80 \%(28)$ & 0.01 \\
\hline
\end{tabular}

LOS length of stay

NS neurosurgery

TSP table of surgical procedure

s.d standard deviation

$B M I$ body-mass index

Unhealthy BMI BMI outside the 18.5-23 range

OT occupational therapy

PT physiotherapy

$H A l$ hospital-acquired infections

Table 3 Stepwise logistic regression on prolonged LOS for HPB sample

\begin{tabular}{|c|c|c|}
\hline Predictors ${ }^{\mathrm{b}}$ & $\operatorname{Exp}(B)$ & $p$-value \\
\hline Constant & 0.01 & 0.002 \\
\hline \multicolumn{3}{|l|}{ Preoperative Factors: } \\
\hline Age & 1.06 & 0.01 \\
\hline Admissions After $5 \mathrm{pm}^{\mathrm{a}}$ & 4.35 & 0.01 \\
\hline
\end{tabular}

LOS length of stay

$H P B$ hepatobiliary surgery

${ }^{a}$ Reference group

${ }^{b}$ Statistically non-significant factors omitted from the table day at the hospital and preventing delays. This is particularly important for the first surgery of the day since delays will cause accumulating scheduling cascades through the day.

The logistic regression model showed that the number of HAI, a postoperative factor, predicted prolonged LOS for the NS sample. This result suggests the importance of reducing surgical site infections and other complications after surgery. Specifically, post-surgical care should focus on wound support and better monitoring by the team for pneumonia and nosocomial infections. Perhaps, hospitals could consider postoperative procedures such 
Table 4 Stepwise logistic regression on prolonged LOS for NS sample

\begin{tabular}{lll}
\hline Predictors $^{\text {b }}$ & Exp(B) & p-value \\
\hline Constant & 0.16 & $<0.001$ \\
Preoperative Factors: & & 0.02 \\
$\quad$ Functional Status & 1.93 & 0.01 \\
$\quad$ Referral to OT & \\
Postoperative Factors: & 7.10 & 0.01 \\
$\quad$ Number of HAl & 19.89 & \\
\hline $\begin{array}{l}\text { LOS length of stay } \\
\text { NS neurosurgery }\end{array}$ & & \\
$\begin{array}{l}\text { OT occupational therapy } \\
\text { HAl hospital-acquired infections } \\
\text { a Reference group } \\
\text { bStatistically non-significant predictors are omitted from the table }\end{array}$
\end{tabular}

as Team Strategies and Tools to Enhance Performance and Patient Safety (TeamSTEPPS) to improve teamwork in patient monitoring among the inpatient care team. Evidence suggests that such protocols can reduce HAI to improve patient safety [55].

A limitation of this study is that the data was hand collected from case notes, which increases the risk of translation error. Random quality checks mitigated this risk. The resource intensity of case notes review meant that we could only collect one complete year of data, which limits the predictive power of our results. A second limitation is the cross-sectional design, which restricts a determination of a causal relationship among the variables. This limitation presents a future research opportunity with longitudinal studies. Finally, our results came from a single site even though the findings are broadly consistent with previous studies in other settings, as noted in the earlier discussion.

\section{Conclusion}

Our findings indicate that preoperative patient factors related to age, functional status, and frailty requiring $\mathrm{OT}$ as well as system factors related to admission after $5 \mathrm{pm}$ prolonged LOS for patients in our HPB and NS samples. Postoperative factor related to the number of HAI was associated with prolonged LOS for the NS sample. The results suggest that patient safety and quality of care may be improved with better presurgery patient preparation and admission practices as well as improved patient monitoring after surgery through better teamwork to reduce HAI. Our results may offer insights for hospital administrators and clinicians to be conscious of patient and system level factors that could lead to prolonged LOS in elective HPB and NS surgeries since no studies have explored perioperative factors for the entire surgical pathway in these two types of high demand elective surgeries. We hope that our findings help hospitals design better patient management services by setting reasonable preoperative expectations for patients and the healthcare team, establishing efficient admission protocols, and providing effective postoperative treatment care that improve meaningful reductions in LOS.

\section{Additional files}

Additional file 1: Median LOS in days according to surgical complexity. (DOCX $19 \mathrm{~kb}$ )

Additional file 2: Coding of the predictor variables. (DOCX $28 \mathrm{~kb}$ )

\section{Abbreviations \\ ASA: American Society of Anesthesiologists; BMl: Body-mass index; ERAS: Enhanced recovery after surgery; HAl: Hospital-acquired infections; HPB: Hepatobiliary; IQR: Interquartile range; LOS: Length of stay; \\ NS: Neurosurgery; OT: Occupational therapy; PT: Physiotherapy; TeamSTEPPS: Team Strategies and Tools to Enhance Performance and Patient Safety; TSP: Table of surgical procedure}

\section{Acknowledgements}

We gratefully acknowledge invaluable help with data acquisition and case reviews from the following.

Normalis Alwi, Asst Director of Nursing*; Wan Ling Chai, Dip (Nurs), RN; Choo Wah Chia, RN, Senior Staff Nurse I*; Jenny Z.Z. Chong, Dip (Nurs), RN+; Alicia A.Y. Chua, Dip (Nurs), RN+; Bingzhen Gao, Dip (Nurs), RN+; Hak See Goh, RM, RN, Senior Staff Nurse I*; Bella P.Y. Hsieh, Dip (Nurs), RN+; Hui Ming Lee, Dip (Nurs), RN+; Sow Kim Lee, BN, RN, Senior Staff Nurse I*; Wai Wai Leong, Dip (Nurs), RN+; Elene J.W. Lim, Dip (Nurs), RN+; Slimbanu d/o Mamat Sardak, RN, Senior Staff Nurse II*; Agnes C.H. Quek, RN, Senior Staff Nurse II*; Fiona Y.H. Tan, Dip (Nurs), RN+; Yee Kian Tay, RN, Senior Staff Nurse I*; Alice Teo, RN, BA, Asst Nurse Clinician*; Sivakami Varadaradjo, Dip (Nurs), RN+; Justyne Y.N. Won, Dip (Nurs), RN+; Yan Hui Xu, BN, RN, Senior Assistant Manager*; Sichun Yang, Dip (Nurs), RN+; Wai Kin Yip, BN, RN, Nurse Educator*

*National University Hospital, Singapore; ${ }^{+}$Ngee Ann Polytechnic Institute.

\section{Funding}

Not applicable

\section{Availability of data and materials}

Mordifi, S Lee, and Seah had full access to all of the data in the study and take responsibility for the integrity of the data and the accuracy of the data analysis.

\section{Previous presentation}

This study has not been previously presented.

\section{Authors' contributions}

Study concept and Design: HSLF, SHL, SYL, SZM. Acquisition, Analysis or Interpretation of Data: SHL, PMYS, JHHT, SZM, AWCK, SL. Drafting of Manuscript: SHL, PHP, JHHT, SZM. Critical Revision of Manuscript for Important Intellectual Content: SHL, PMYS, SYL, PHP, SZM. Statistical Analysis: SHL. Administrative Technical or Material Support: JHHT, PMYS, SYL, SZM, AWCK, SL. Study Supervision: HSLF, SYL, SZM. All authors read and approved the final manuscript.

\section{Ethics approval and consent to participate}

This research was approved by the National Healthcare Group's Domain Specific Review Board, Study \#: 2015/00229, on April 7, 2015. This study draws on de-identified archival records and there was no requirement to obtain consent to participate.

Consent for publication

Not applicable 


\section{Competing interests}

Siu Yin Lee, Soo-Hoon Lee, Jenny H.H. Tan, Howard S.L. Foo, Phillip H. Phan, Alfred W.C. Kow, Sein Lwin, Penelope M.Y. Seah, Siti Zubaidah Mordiffi report no relevant financial interests, activities, relationships, and affiliations competing of interests.

\section{Publisher's Note}

Springer Nature remains neutral with regard to jurisdictional claims in published maps and institutional affiliations.

\section{Author details}

${ }^{1}$ National University Hospital, 5 Lower Kent Ridge Road, Singapore 119074, Singapore. ${ }^{2}$ Old Dominion University, 1 Old Dominion University, Norfolk, VA 23529, USA. ${ }^{3}$ Ngee Ann Polytechnic, 535 Clementi Road, Singapore 599489, Singapore. ${ }^{4}$ Woodlands Health, 9 Maxwell Road, Singapore 069112 , Singapore. ${ }^{5}$ Johns Hopkins University, 100 International Drive, Baltimore, MD 21202, USA.

Received: 17 January 2017 Accepted: 20 December 2017 Published online: 05 January 2018

\section{References}

1. Kalish RL, Daley J, Duncan CC, Davis RB, Coffman GA, lezzoni LI. Costs of potential complications of care for major surgery patients. Am J Med Quality. 1995; 10:48-54.

2. Collins TC, Daley J, Henderson WH, Khuri SF. Risk factors for prolonged length of stay after major elective surgery. Ann Surg. 1999;230(2):251-9.

3. De Coster C, Kozyrskyj A. Long-stay Patients in Winnipeg Acute Care Hospitals. Winnipeg, MB: Manitoba Centre for Health Policy and Evaluation; 2000.

4. Singer AJ, Thode HC Jr, Viccellio P, Pines JM. The association between length of emergency department boarding and mortality. Acad Em Med. 2011;18(12):1324-9.

5. Schneider $\mathrm{EB}$, Hyder $\mathrm{O}$, Wolfgang $\mathrm{CL}$, et al. Patient readmission and mortality after surgery for hepato-pancreato-biliary malignancies. J Am Coll Surg. 2010;215(5):607-15

6. Jannasch $\mathrm{O}$, Kelch B, Adolf D, et al. Nosocomial infections and microbiologic spectrum after major elective surgery of the pancreas, liver, stomach, and esophagus. Surg Infect. 2015;16(3):338-45.

7. Freitas A, Silva-Costa T, Lopes F, et al. Factors influencing hospital high length of stay outliers. BMC Health Serv Res. 2012;12:265.

8. Haider AH, Obirieze A, Velopulos CG, et al. Incremental cost of emergency verus elective surgery. Ann Surg. 2015;262(2):260-6.

9. Macario A, Vitez TS, Dunn B, McDonald T, Brown B. Hospital costs and severity of illness in three types of elective surgery. Anesthesiology. 1997;86:92-100.

10. Pattakos G. Predicting length of stay and non-home discharge: a novel approach to reduce wasted resources after cardiac surgery. 2011.(Electronic Thesis or Dissertation). Retrieved from https://etd.ohiolink.edu/pg_10?0::NO: 10:P10_ACCESSION NUM:case1291145768. Accessed 26 May 2017.

11. Osnabrugge RL, Speir AM, Head SJ, et al. Prediction of costs and length of stay in coronary artery bypass grafting. Ann Thorac Surg. 2014;98(4):1286-93.

12. Greenblatt DY, Kelly K, Rajamanickam V, et al. Preoperative factors predict perioperative morbidity and mortality after pancreaticoduodenectomy. Ann Surg Oncol. 2011;18(8):2126-35.

13. Morimoto $Y$, Mizuno $H$, Akamaru $Y$, et al. Predicting prolonged hospital stay after laparoscopic cholecystectomy. Asian J Endosc Surg. 2015;8(3):289-95.

14. Tsang YY, Poon CM, Lee KW, Leong HT. Predictive factors of long hospital stay after laparoscopic cholecystectomy. Asian J Surg. 2007;30(1):23-8.

15. Seicean A, Alan N, Seicean $S$, et al. Impact of increased body mass index on outcomes of elective spinal surgery. Spine. 2014;39(18):1520-30.

16. Gruskay JA, Fu M, Bohl DD, Webb ML, Grauer JN. Factors affecting length of stay after elective posterior lumbar spine surgery: a multivariate analysis. Spine J. 2015;15(6):1188-95.

17. Poon D, Anderson BO, Chen LT, et al. Management of hepatocellular carcinoma in Asia: consensus statement from the Asian oncology summit 2009. Lancet Oncol. 2009;10:1111-8.

18. Wong TCL, Poon RTP. Hepatobiliary malignancies: lessons from Asia. Dig Dis. 2013;31:130-7.

19. Wong JS, Ng KH, Wong SH. Intracranial aneurysms in Sarawak general hospital over a 30-month period. J Clin Neurosci. 2004;11(3):254-8.
20. Almashrafi A, Elmontsri M, Aylin P. Systematic review of factors influencing length of stay in ICU after adult cardiac surgery. BMC Health Serv Res. 2016;16:318

21. Lukasiewicz AM, Grant RA, Basques BA, Webb ML, Samuel AM, Grauer JN. Patient factors associated with 30-day morbidity, mortality, and length of stay after surgery for subdural hematoma: a study of the American College of Surgeons National Surgical Quality Improvement Program. J Neurosurg. 2016;124(3):760-6.

22. Sacks GD, Lawson EH, Sawes AJ, Gibbons MM, Zingmond DS, Ko CY. Which patients require more care after hospital discharge? An analysis of post-acute care use among elderly patients undergoing elective surgery. J Am Coll Surg 2015;220(6):1113-21.

23. Chang JK, Calligaro KD, Lombardi JP, Dougherty MJ. Factors that predict prolonged length of stay after aortic surgery. J Vasc Surg. 2003;38:335-9.

24. Cannistrà M, Grande R, Ruggiero M, et al. Resection of hepatocellular carcinoma in elderly patients and the role of energy balance. Int J Surg. 2016:33:S119-25.

25. Sundaram V, Lim J, Tholey DM, et al. The Braden scale, a standard tool for assessing pressure ulcer risk, predicts early outcomes after liver transplantation. Liver Transpl. 2017; https://doi.org/10.1002/lt.24789.

26. Lilamand M, Kelaiditi E, Cesari M, Raynaud-Simon A, Ghisolfi A, Guyonnet S, Vellas B, van Kan GA. Toulouse frailty platform team, validation of the mini nutritional assessment-short form in a population of frail elders without disability. J Nutr Health Aging. 2013;19:570-4.

27. McGirt MJ, Parker SL, Chotai S, et al. Predictors of extended length of stay, discharge to inpatient rehab, and hospital readmission following elective lumbar spine surgery: introduction of the Carolina-Semmes grading scale. J Neurosurg Spine. 2017;12:1-9.

28. Partridge JSL, Fuller M, Harari D, Taylor PR, Martin FC, Dhesi JK. Frailty and poor functional status are common in arterial vascular surgical patients and affect postoperative outcomes. Int J Surg. 2015;18:57-63.

29. Matsui K, Goldman L, Johnson PA, Kuntz KM, Cook EF, Lee TH. Comorbidity as a correlate of length of stay for hospitalized patients with acute chest pain. J Gen Intern Med. 1996;11(5):262-8.

30. Yip WK, Mordiffi SZ, Wong HC, Ang ENK. Development and validation of a simplified falls assessment tool in an acute care setting. J Nurs Care Qual. 2016;31(4):310-7.

31. Oliver D, Healey F, Haines TP. Preventing falls and fall-related injuries in hospitals. Clin Geriatr Med. 2010;26(4):645-92.

32. Kronzer $V L$, Jerny $M R$, Abdallah $A B$, et al. Preoperative falls predict postoperative falls, functional decline, and surgical complications. EBioMedicine. 2016;12:302-8.

33. Fransen M. When is physiotherapy appropriate? Best Pract Res Clin Rheumatol. 2004;18:477-89.

34. American Occupational Therapy Association. Definition of occupational therapy practice for state regulation. Policy 5.3.1. Am J Occup Ther. 1994;48:1072-3.

35. Polastri M, Dell'Amore A, Zagnoni G, Nava S. Preoperative physiotherapy in subjects with idiopathic pulmonary fibrosis qualified for lung transplantation: implications on hospital length of stay and clinical outcomes. J Thorac Dis. 2016;8(5):E264-8.

36. Lim SC, Doshi V, Castasus B, Lim JKH, Mamun K. Factors causing delay in discharge of elderly patients in an acute care hospital. Ann Acad Med Singap. 2006;35(1):27-32.

37. Earnest A, Chen MIC, Seow E. Exploring if day and time of admission is associated with average length of stay among inpatients from a tertiary hospital in Singapore: an analytic study based on routine admission data. BMC Health Serv Res. 2006;6:6.

38. Attenello FJ, Wen T, Cen SY, et al. Incidence of "never events" among weekend admissions versus weekday admissions to US hospitals: national analysis. BMJ. 2015;350:h1460. https://doi.org/10.1136/bmj.h1460.

39. Wong C, Yu R. Preoperative preparation for pheochromocytoma resection: physician survey and clinical practice. Exp Clin Endocrinol Diabetes. 2010; 118(7):400-4.

40. Nobili A, Licata G, Salerno F, et al. Polypharmacy, length of hospital stay, and in-hospital mortality among elderly patients in internal medicine wards. The REPOSI study. Eur J Clin Pharmacol. 2011;67(5):507-19.

41. Arnold PM, Rice LR, Anderson KK, McMahon JK, Connelly LM, Norvell DC. Factors affecting hospital length of stay following anterior cervical discectomy and fusion. Evid Based Spine Care J. 2011;2(3):11-8.

42. Mortazavi SS, Shati M, Keshtkar A, Malakouti SK, Bazargan M, Assari S. Defining polypharmacy in the elderly: a systematic review protocol. BM Open. 2016;6:e010989. https://doi.org/10.1136/bmjopen-2015-010989. 
43. Miller AC, Polgreen LA, Cavanaugh JE, Polgreen PM. Hospital clostridium difficile infection rates and prediction of length of stay in patients without C. Difficile infection. Infect Control Hosp Epidermiol. 2016;37(4):404-10.

44. Reponen E, Korja M, Niemi T, Silvasti-Lundell M, Hernesniemi J, Tuominen H. Preoperative identification of neurosurgery patients with a high risk of inhospital complications: a prospective cohort of 418 consecutive elective craniotomy patients. J Neurosurg. 2015;123(3):594-604.

45. Kable AK, Gibberd RW, Spigelman AD. Adverse events in surgical patients in Australia. Int J Qual Health Care. 2002;14(4):269-76.

46. Zegers $M$, de Bruijne MC, de Keizer B, et al. The incidence, root-causes, and outcomes of adverse events in surgical units: implication for potential prevention strategies. Patient Saf Surg. 2011;5:13. https://doi.org/10.1186/ 1754-9493-5-13

47. United Nations, Department of Economic and Social Affairs, Population Division (2015). World population ageing 2015 (ST/ESA/SER.A/390),

48. Table of Surgical Procedures, Ministry of Health. Accessed 3 May 2016 from https://www.moh.gov.sg/content/dam/moh_web/Healthcare_Financing/ Medisave/TOSP\%20Booklet\%20with\%20MSP\%20(3\%20Jan\%202017).pdf. Accessed 26 Dec 2017

49. Bergstrom N, Braden BJ, Laguzza A, Holman V. The Braden scale for predicting pressure sore risk. Nurs Res. 1987;36:205-10.

50. Lim SL, Ong KCB, Chan YH, Loke WC, Ferguson M, Daniels L. Malnutrition and its impact on cost of hospitalization, length of stay, readmission and 3-year mortality. Clin Nutr. 2012:31(3):345-50.

51. Katz S, Ford AB, Moskowitz RW, Jackson BA, Jaffe MW. Studies of illness in the aged: the index of ADL: a standardized measure of biological and psychosocial function. JAMA. 1963;185(12):914-9.

52. IBM Corp. Released 2015. IBM SPSS Statistics for Windows, Version 23.0. Armonk, NY: IBM Corp.

53. Wind J, Polle SW, Kon F, PHP J, et al. Systematic review of enhanced recovery programmes in colonic surgery. Br J Surg. 2006;93(7):800-9

54. Coolsen MM, van Dam RM, van der Wilt AA, Slim K, Lassen $\mathrm{K}$, Dejong CH. Systematic review and meta-analysis of enhanced recovery after pancreatic surgery with particular emphasis on pancreaticoduodenectomies. World J Surg. 2013;37(8):1909-18.

55. Weaver SJ, Rosen MA, DiazGranados D, et al. Does teamwork improve performance in the operating room? A multilevel evaluation. Jt Comm J Qual Patient Saf. 2010;36(3):133-42.

\section{Submit your next manuscript to BioMed Central and we will help you at every step:}

- We accept pre-submission inquiries

- Our selector tool helps you to find the most relevant journal

- We provide round the clock customer support

- Convenient online submission

- Thorough peer review

- Inclusion in PubMed and all major indexing services

- Maximum visibility for your research

Submit your manuscript at www.biomedcentral.com/submit

) Biomed Central 